\title{
Observation of orientational influences on vibrational energy transfer at metal surfaces: Rotational cooling associated with vibrational relaxation at a metal surface
}

\author{
Alec M. Wodtke ${ }^{\mathrm{a}, *}$, Huang Yuhui ${ }^{\mathrm{b}}$, Daniel J. Auerbach ${ }^{\mathrm{c}}$ \\ a Department of Chemistry and Biochemistry, University of California Santa Barbara, 4623C Physical Sciences North, \\ Santa Barbara, CA 93106, United States \\ b Oplink Communications, 3469 North First Street, San Jose, CA 95134-1803, United States \\ ${ }^{\mathrm{c}}$ Hitachi Global Storage Technologies, 650 Harry Road, San Jose, CA 95120-6099, United States
}

Received 14 June 2005

Available online 2 September 2005

\begin{abstract}
The degree of rotational excitation resulting from collisions of $\mathrm{NO}(v=0)$ and $\mathrm{NO}(v=2)$ with a $\mathrm{Au}(111)$ surface is smaller for $\mathrm{NO}(v=2)$ that undergoes vibrational relaxation, $\mathrm{NO}(v=2 \rightarrow v=1)$, than it is for $\mathrm{NO}$ that scatters in a vibrationally elastic channel, $\mathrm{NO}(v=2 \rightarrow v=2)$ or $\mathrm{NO}(v=0 \rightarrow v=0)$. The rotational excitation is less for the vibrational relaxation channel, despite the fact that vibrational relaxation releases $0.25 \mathrm{eV}$ that is potentially available for increased rotational excitation. This difference in amount of rotational excitation for vibrationally inelastic and elastic channels increases with the kinetic energy, $E_{\mathrm{i}}$, of the incident NO molecules. These observations should provide useful comparisons to full dimensional theories of surface interactions.

(C) 2005 Elsevier B.V. All rights reserved.
\end{abstract}

\section{Introduction}

Mechanisms of vibrational energy exchange between molecules and metal surfaces fall into two general categories, electronically adiabatic (i.e., mechanical) and electronically non-adiabatic. Mechanical mechanisms describe the energy transfer in terms of the changes in the force field experienced by the molecule during the surface collision. For example, it has been suggested that trajectories that pass near the $\mathrm{H}-\mathrm{H}$ dissociative-adsorption transition state are responsible for conversion of translational energy of incidence to vibrational excitation when $\mathrm{H}_{2}$ scatters from copper [1]. Similar 'bond softening' ideas have been used to explain vibrational excitation of NO in collisions with silver surfaces [2-4]. Electronically non-adiabatic mechanisms $[5,6]$ describe

\footnotetext{
${ }^{*}$ Corresponding author. Fax: +1 8058934120.

E-mail address: wodtke@chem.ucsb.edu (A.M. Wodtke).
}

transfer of vibrational energy to create excited electron hole pairs (EHPs) in the metal and vice-versa vibrational excitation of molecules by relaxation of thermally excited metallic EHPs $[7,8]$.

A great deal of experimental work in this area has been dedicated to uncovering the relative importance of mechanical vs. electronically non-adiabatic mechanisms [9-11]. There has been less attention paid to addressing the likely inadequacies of reduced dimensionality models which dominate the theoretical landscape in this field. For a diatom (A-B) colliding with a surface, molecule-surface distance $(z)$, A-B bond distance $(R)$, site of attack at the surface $(x, y)$ and $\mathrm{A}-\mathrm{B}$ bond orientation with respect to surface normal $(\theta, \varphi)$ are all potentially important degrees of freedom. Early theories of vibrational energy transfer were 1-D $(z)$ in nature [5], while more modern approaches have extended to 2-D $(z, R)$ [12]. Only a few examples can be found that have attempted to include additional 
degrees of freedom $[13,14]$. As far as experiment is concerned, there are few if any results which have been shown to probe the angular and or site specific aspects of the factors controlling vibrational energy exchange at metal surfaces. Experimental results like this would provide valuable benchmarks for testing more sophisticated multidimensional theories of energy transfer at surfaces.

We have previously reported on aspects of the scattering dynamics of vibrationally excited $\mathrm{NO}(v=2)$ from a $\mathrm{Au}(111)$ surface, including the incidence energy dependence of survival probability [10], the surface temperature dependence of vibrational excitation probability [11] and the insensitivity of trapping to NO vibration [15]. Here, we present data on the rotational excitation associated with vibrationally inelastic events at metal surfaces. We find the counterintuitive result that those molecules which undergo vibrational relaxation (releasing $0.25 \mathrm{eV}$ per vibrational quantum) can emerge from the surface scattering event with a reduced degree of rotational excitation compared to molecules that undergo vibrationally elastic collisions. The degree of 'rotational cooling associated with vibrational relaxation' is seen to increase with incidence energy. This work provides a direct experimental observation of the influences of angular anisotropies and site specific influences $(\theta, \varphi, x, y)$ important for vibrationally inelastic collisions at metal surfaces. As such, they provide a new benchmark for comparison with theories of vibrational energy exchange presently under development.

\section{Experimental}

The experimental arrangement has been described previously in detail $[9-11,15,16]$. In these experiments $\mathrm{NO}(v=2, J=1.5, \Omega=0.5)$ in both elf parity states was prepared by infrared overtone pumping of a supersonic beam of NO $15-\mathrm{cm}$ upstream from the surface. For this, we generated $2.7 \mu \mathrm{m}\left(3729 \mathrm{~cm}^{-1}\right)$ light $\left(0.08 \mathrm{~cm}^{-1}\right.$ linewidth, $\sim 6 \mathrm{~mJ} /$ pulse, $5 \mathrm{~ns}$ pulse duration $)$ by mixing $120 \mathrm{~mJ} /$ pulse of $761.8 \mathrm{~nm}$ light from a $\mathrm{Nd}$ :YAG pumped dye laser (operating on LDS-765) with the $1.064 \mu \mathrm{m}$ fundamental of the Nd:YAG laser in a $\mathrm{LiNbO}_{3}$ crystal. We estimate $\sim 2 \%$ of the total population in the illuminated part of the molecular beam is pumped to the desired state.

The supersonic NO molecular beam was generated using a pulsed molecular beam source [17], collimated by passage through three differentially pumped stages, and impinged at normal incidence upon a $\mathrm{Au}\left(\begin{array}{lll}1 & 1 & 1\end{array}\right)$ crystal mounted in an ultra-high-vacuum $\left(<10^{-9}\right.$ Torr $)$ chamber. Seeding $\mathrm{NO}$ in $\mathrm{He}$ and $\mathrm{H}_{2}$ allowed us to vary the incidence energy of the NO.

The $A u(111)$ surface was cleaned daily by sputtering with $2.8 \mathrm{kV} \mathrm{Ar}{ }^{+}$for $30 \mathrm{~min}$. and flash annealing to
$1100 \mathrm{~K}$. After $9 \mathrm{~h}$ exposure to the NO beam, surface contamination was still below the detection limit of $\mathrm{Au}$ ger electron spectroscopy (AES). Even so, we annealed the surface between each experimental run (approximate every $2 \mathrm{~h}$ ).

We detected either the laser-prepared state of NO or states resulting from scattering at the surface with onecolor, two-photon REMPI through the $\mathrm{A}^{2} \sum^{+} \leftarrow \mathrm{X}^{2} \Pi_{i}$ $\gamma$-bands. The doubled output from a frequency tripled Nd:YAG laser (Continuum 7010) pumped a Lambda Physik FL3002 dye laser operating on Coumarin 480 and 503 was used to probe the $\gamma(0,1), \gamma(0,2)$ and $\gamma(0,3)$ bands for NO scattered in $v=1,2$ and 3 levels, respectively. One advantage of this detection scheme is that there is no spectral interference, which was critical when using a relatively low resolution UV laser $\left(0.5 \mathrm{~cm}^{-1}\right.$ bandwidth) which was available for these experiments. All transition frequencies were calibrated against the calculated values obtained from term energies of $\mathrm{X}$ and A states of NO using literature values for spectroscopic constants [18-20]. The REMPI signal power dependence was measured to be linear even though a low power unfocused probe laser beam was used. The size of the laser beam was enlarged and the distance from the surface was minimized to ensure that the experiment reflected the angle integrated signal.

For comparison, the vibrationally inelastic scattering of $\operatorname{NO}(v=0)$ was also investigated. Here, scattered products were detected by REMPI through of the $\gamma(1,0)$ band. The laser system employed for the overtone pumping was changed to a dual 2400 grating configuration and used to generation $214 \mathrm{~nm}$ light by frequency tripling in BBO. This setup produced identical amount of probe laser power in the entire spectral region of interested with bandwidth of $\sim 0.10 \mathrm{~cm}^{-1}$.

\section{Results}

Fig. 1 shows a typical REMPI spectrum of NO scattered into $v=1$ when an optically prepared beam containing $\mathrm{NO}$ in $v=2$ with a mean kinetic energy $E_{\mathrm{i}}=63 \mathrm{~kJ} / \mathrm{mol}$ is incident on the $\mathrm{Au}(111)$ surface at room temperature. Both pump and probe laser power were recorded and used to normalize the spectrum. When the pump laser was blocked, those REMPI signals arising from transitions in the $\gamma(0,2)$ and $\gamma(0,3)$ bands disappeared entirely, a few percent of the signal remained for the $\gamma(0,1)$ band because of very small effusive part $(v=1)$ in the beam and vibrational excitation of ground state NO scattered from the surface, etc. This became a severe problem with increasing surface temperature because of exponentially increasing in vibrational excitation probability. Therefore, for rotational temperature determinations in $v=1$ state, the final REMPI spectrum was obtained from direct subtraction of 


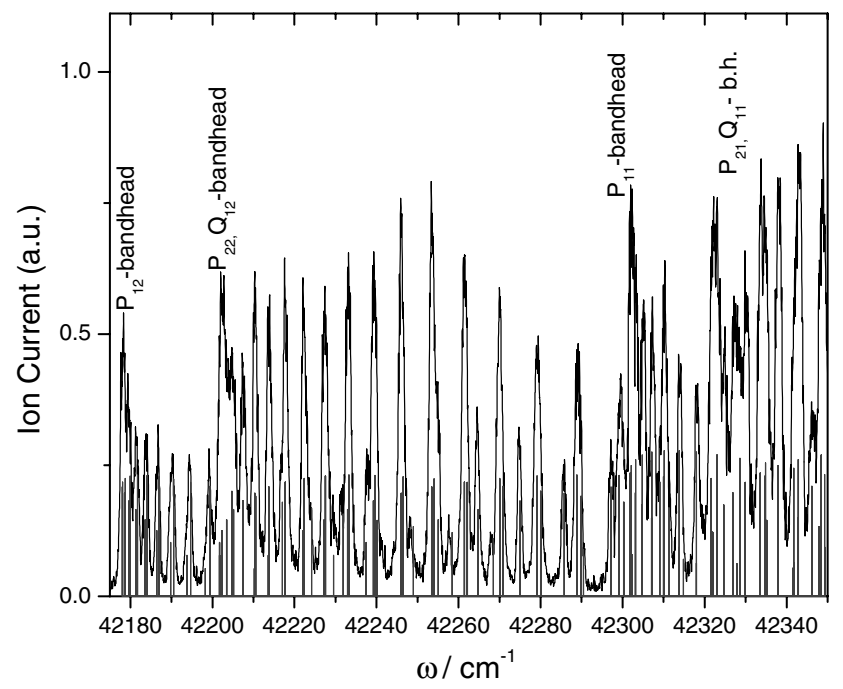

Fig. 1. Rotationally resolved REMPI spectrum of $\mathrm{NO}(v=1)$ molecules that are produced by vibrational relaxation of $\mathrm{NO}(v=2)$ in collisions with $\mathrm{Au}(111)$. The incidence energy in this experiment was $63 \mathrm{~kJ} / \mathrm{mol}$ and the surface temperature was $300 \mathrm{~K}$. The stick spectrum was calculated (assuming $T_{\text {rot }}=1000 \mathrm{~K}$ ) from spectroscopic theory of the ${ }^{2} \sum^{+}(v=0) \leftarrow{ }^{2} \Pi(v=1)$, that is the $\gamma(0,1)$ band [18-20]. Four prominent bandheads are highlighted.

two spectra recorded with and without the IR pump laser using the toggle mode on an SRS boxcar.

The rotational temperature for each vibrational level was determined with two methods: (1) Boltzmann plots of individual branches of the A-X band used and (2) the least squares global fit to the entire spectrum using a homemade Mathematica program.

Table 1 gives a summary of these determinations of NO rotational temperature for the vibrational channels $\mathrm{NO}(v=2 \rightarrow v=2), \quad \mathrm{NO}(v=2 \rightarrow v=1), \quad \mathrm{NO}(v=2 \rightarrow$

Table 1

Summary of the rotational temperature determination of NO scattered under different kinetic energies, $E_{\mathrm{i}}$, and surface temperatures, $T_{\mathrm{s}}$

\begin{tabular}{lllcc}
\hline$E_{\mathrm{i}}(\mathrm{kJ} / \mathrm{mol})$ & $V_{\text {in }}$ & $V_{\text {fin }}$ & $T_{\text {rot }}\left(T_{\mathrm{s}}=298 \mathrm{~K}\right)$ & $T_{\text {rot }}\left(T_{\mathrm{s}}=480 \mathrm{~K}\right)$ \\
\hline 5.3 & 2 & 2 & $277 \pm 20$ & $334 \pm 40$ \\
5.3 & 0 & 0 & $244 \pm 30$ & $313 \pm 60$ \\
$\mathbf{5 . 3}$ & $\mathbf{2}$ & $\mathbf{1}$ & $\mathbf{2 4 0} \pm \mathbf{3 0}$ & \\
8.7 & 0 & 0 & $404 \pm 40$ & $474 \pm 25$ \\
8.7 & 2 & 2 & $363 \pm 20$ & $362 \pm 50$ \\
$\mathbf{8 . 7}$ & $\mathbf{2}$ & $\mathbf{1}$ & $\mathbf{3 1 0} \pm \mathbf{3 0}$ & \\
29 & 0 & 0 & $727 \pm 100$ & $782 \pm 50$ \\
29 & 2 & 2 & $777 \pm 100$ & $1013 \pm 200$ \\
$\mathbf{2 9}$ & $\mathbf{2}$ & $\mathbf{1}$ & $\mathbf{5 7 6} \pm \mathbf{7 4}$ & $\mathbf{6 4 4} \pm \mathbf{9 0}$ \\
63 & 0 & 0 & $1250 \pm 120$ & $1284 \pm 80$ \\
63 & 2 & 2 & $1160 \pm 200$ & $1483 \pm 180$ \\
$\mathbf{6 3}$ & $\mathbf{2}$ & $\mathbf{1}$ & $\mathbf{1 0 4 0} \pm \mathbf{1 4 0}$ & $\mathbf{1 1 6 6} \pm \mathbf{2 0 0}$ \\
63 & 2 & 3 & & $652 \pm 25$ \\
72 & 2 & 2 & $1439 \pm 200$ & \\
72 & 0 & 0 & $1545 \pm 250$ & $1545 \pm 350$ \\
\hline
\end{tabular}

Here, $V_{\text {in }}$ and $V_{\text {fin }}$ represent the vibrational states prepared and probed, respectively. Vibrationally sub-elastic processes are shown in bold font, vibrationally super-elastic in italics. $v=3)$, and $\mathrm{NO}(v=0 \rightarrow v=0)$ for surface temperatures of 298 and $480 \mathrm{~K}$ and mean collisional energies from $5.3 \mathrm{~kJ} / \mathrm{mol}$ to $72 \mathrm{~kJ} / \mathrm{ml}$. The final rotational state distribution is virtually independent of the surface temperature, $T_{\mathrm{s}}$, but strongly depends on the incident energy, $E_{\mathrm{i}}$. The rotational temperature of the products is colder than the surface temperature when $\mathrm{NO}$ with $E_{\mathrm{i}}=8.7 \mathrm{~kJ} /$ mol was introduced to the $\mathrm{Au}(111)$ at $T_{\mathrm{s}}=480 \mathrm{~K}$. These results are consistent with a direct inelastic scattering mechanism [5-7]. A weak rotational rainbow was observed in the spectra for $J \geqslant 32.5$ when the molecular beam with the highest mean translational energy was used in the experiments. Although a Boltzmann distribution is not an entirely accurate description of the the observed rotational state distributions, it is a reasonably good approximation for these spectra. This approximation did increase the error bars on our determination of rotational excitation.

Vibrational excitation $(v=3)$ was unambiguously observed in the molecular scattering of $\operatorname{NO}(v=2) /$ $\mathrm{Au}(111)$ at higher surface temperature with higher collisional energy. However, due to an experimental lack of sensitivity, no evidence for vibrational excitation could be found for NO with $E_{\mathrm{i}}=8.7 \mathrm{~kJ} / \mathrm{mol}$ and $T_{\mathrm{s}}<480 \mathrm{~K}$. However, excitation efficiency was determined to be $\sim 0.08 \%$ for $\mathrm{NO}(v=2)$ with $E_{\mathrm{i}}=63 \mathrm{~kJ} / \mathrm{mol}$ even on a room temperature surface. The $\mathrm{NO}(v=3) / \mathrm{NO}(v=2)$ ratios and vibrational excitation probabilities discussed in the following paragraph could be fitted into an Arrhenius equation of $\left(-E_{\mathrm{a}} / R T_{\mathrm{s}}\right)$ [5-7] in lower surface temperature part for each collisional energy, where $E_{\mathrm{a}}$ was determined to be $24 \pm 3 \mathrm{~kJ} / \mathrm{mol}$, the energy difference between two vibrational states. The pre-exponential, A, was found to depend much more strongly on incident energy than those in $\mathrm{NO} / \mathrm{Cu}\left(\begin{array}{lll}1 & 1 & 0\end{array}\right)$ and $\mathrm{NO} / \mathrm{Ag}\left(\begin{array}{lll}1 & 1 & 1\end{array}\right)$ systems [5-7], about 10 times larger for $\mathrm{NO}$ with $E_{\mathrm{i}}=63 \mathrm{~kJ} / \mathrm{mol}$ than that of $E_{\mathrm{i}}=29 \mathrm{~kJ} / \mathrm{mol}$. The rotational temperature for $\mathrm{NO}(v=3)$ was $652 \pm 25 \mathrm{~K}$ for $T_{\mathrm{s}}=480 \mathrm{~K}$ and $E_{\mathrm{i}}=63 \mathrm{~kJ} / \mathrm{mol}$. No rotational rainbow could be identified from the spectrum. These are consistent with previous experimental findings obtained from $\mathrm{NO} / \mathrm{Ag}(1111)[5,6]$ and $\mathrm{NO} / \mathrm{Cu}(110)$ [7].

Inspection of Table 1 reveals that for each 'vibrational triplet' of measurements, the vibrationally subelastic channel $(v=2 \rightarrow 1)$ results in a reduced $T_{\text {rot }}$ compared to the vibrationally elastic channels. For the one measurement of a vibrationally super-elastic channel $(v=2 \rightarrow 3)$, a reduced $T_{\text {rot }}$ is also observed. Thus, the vibrationally inelastic processes all exhibit less rotational excitation than their vibrationally elastic analogs. While this would not perhaps be surprising for vibrationally super-elastic collisions as one might argue that some translational energy of incidence is channeled into vibration of the outgoing molecule, an analogous explanation for sub-elastic collisions seems quite unlikely. 


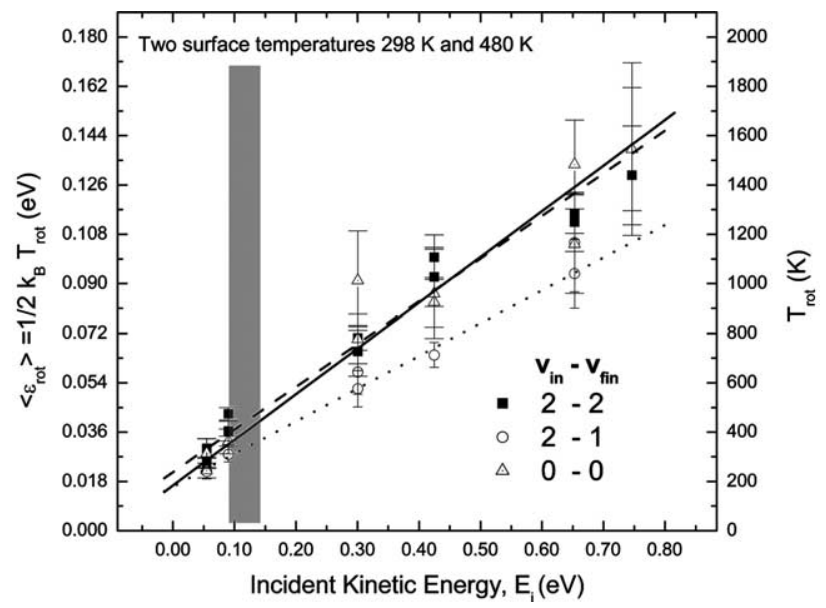

Fig. 2. Efficiency for conversion of translational energy of incidence to final rotational energy. The derived $T_{\text {rot }}$ is shown for six translational energies of incidence and three vibrational channels, $v=2 \rightarrow 2$, $v=2 \rightarrow 1$ and $v=0 \rightarrow 0$. In comparison to vibrationally elastic channels, the vibrationally inelastic channel exhibits reduced rotational excitation as incidence energy is increased. The gray bar $(\sim 0.1 \mathrm{eV})$ indicates the 1/e incidence energy of translation for NO trapping at the surface [15]. Above this energy, direct-scattering dominates.

Table 2

Incidence energy dependence of rotational excitation, $\left\langle E_{\text {rot }}\right\rangle=\alpha\left(E_{\mathrm{i}}+\varepsilon\right)$

\begin{tabular}{lll}
\hline Channel $\left(v_{\text {in }}-v_{\text {fin }}\right)$ & $\alpha$ & $\varepsilon(\mathrm{eV})$ \\
\hline $0-0$ & $0.165 \pm 0.012$ & 0.10 \\
$2-2$ & $0.154 \pm 0.008$ & 0.14 \\
$2-1$ & $0.118 \pm 0.011$ & 0.14 \\
\hline
\end{tabular}

The principle result of this work can be seen most easily in Fig. 2, a graphical representation of Table 1. Here, the average rotational excitation of scattered NO is plotted for a series of experiments carried out at different incidence energies $E_{\mathrm{i}}$. The average rotational energy represented by a rotational distribution at $T_{\text {rot }}$ is given by $\left\langle\varepsilon_{\mathrm{rot}}\right\rangle=1 / 2 k_{\mathrm{B}} T_{\text {rot }}$, where $k_{\mathrm{B}}$ is the Boltzmann constant. The dependence of $\left\langle\varepsilon_{\text {rot }}\right\rangle$ on incidence energy can be fitted empirically to a straight line as in prior work [21-23]. While the intercept $(\varepsilon)$ has not been shown to have an unambiguous physical interpretation [22,23], the slope $(\alpha)$ indicates the efficiency for converting translational energy of incidence to rotational energy of the scattered molecules [21].

These parameters are collected in Table 2 for the three of the scattering channels observed here, $v=2 \rightarrow 2, v=2 \rightarrow 1$ and $v=0 \rightarrow 0$. One can see that the two vibrationally elastic channels exhibit an $\alpha$-value nearly $50 \%$ larger than the vibrationally inelastic channel. Furthermore, this indicates that for collisions in the upper range of incidence energies studied here, molecules that have undergone vibrational relaxation releasing $0.25 \mathrm{eV}$ of energy in a surface collision are emerging from the surface with less rotational energy than mole- cules that exchange no vibrational energy. This observation represents an interesting qualitative behavior that has not as yet been captured in any theoretical treatment of energy transfer at surfaces.

\section{Discussion}

In considering possible explanations for the observed behavior, it is essential first to consider the possible influence of a change in scattering mechanism over the range of translational incidence energies, $E_{\mathrm{i}}$, probed here. If the vibrational energy transfer mechanism were qualitatively different at low and high values of $E_{\mathrm{i}}$ it might not be surprising to observe changing dynamics as a function of $E_{\mathrm{i}}$. Specifically, one expects trapping/ desorption to dominate at low $E_{\mathrm{i}}$, while direct-scattering dominates at high $E_{\mathrm{i}}$.

We have recently reported a detailed analysis of the trapping of $\mathrm{NO}$ on $\mathrm{Au}(111)$ and derived its dependence on translational and vibrational energy of incidence [15]. In that work, no influence of initial vibrational state on trapping could be observed up to $v=2$. In contrast, the dependence on $E_{\mathrm{i}}$ was very well approximated by an exponential decay with a $1 / e$ value of $0.09 \mathrm{eV}$. This is indicated in Fig. 2 by the gray bar. This result shows that while trapping of NO to the Gold surface is significant at the lowest incidence energy shown in Fig. 2, by the time $E_{\mathrm{i}}$ is greater than about $0.1 \mathrm{eV}$, trapping is unimportant [15]. Thus, we attribute the decreased efficiency of translational to rotational energy transfer for vibrationally inelastic scattering as a dynamical effect associated solely with the direct inelastic scattering.

Previous work has yielded strong evidence that the vibrationally inelastic processes of $\mathrm{NO}$ on Gold are strongly influenced by electronically non-adiabatic ( $v \leftrightarrow$ EHP) effects [9-11]. Thus, the observed rotational cooling with vibrational relaxation appears to offer insights into the dynamics of electronically non-adiabatic energy transfer. We favor this conclusion also in view of the characteristic of mechanical mechanisms, where there is a tendency to exchange energy between vibration and translation/rotation of the scattering molecule $[2,4]$. Hence, when a molecule undergoes vibrational relaxation by collision at a surface, mechanical models predict a substantial fraction of the lost vibrational energy to appear as molecular rotation and translation of the scattered molecules. In contrast, we observe a reduced degree of rotational excitation when compared to vibrationally elastic interactions.

Correlations between kinetic energy transfer to rotation and to phonons have been reported in collisions of NO with $\operatorname{Ag}(111)$ [24,25]. Here, a smaller coupling of kinetic energy to phonon excitation was observed for NO molecules which had a larger degree of rotational excitation. The explanation for this anticorrelation of 
energy transfer to phonons and rotation involved a common stochastic variable that mediates both rotational excitation and the phonon excitation. This variable was the orientation of the NO molecules as they collide with the surface. NO which collides nearly vertically or horizontally, will undergo smaller rotational excitation but produce large phonon excitation; NO which collides with the surface with its axis inclined will undergo larger rotational excitation and produce. smaller phonon excitation.

A similar effect may be involved in the results reported here. There may an orientation-dependence or site-dependence $(\theta, \varphi, x, y)$ of the electronically non-adiabatic couplings. Electron Friction with Molecular Dynamics is one of the only methods where attempts have been made to explicitly include the influences of molecular orientation on vibrational energy transfer (see [6] and references therein). In this approach, energy transfer to and from EHP's is modeled as a frictional force. Electronic friction constants can be calculated from $\mathrm{ab}$ initio electronic structure theory and depend on the molecular coordinates. For example, if the electronic friction was stronger for collisions where NO is nearly horizontal or vertical, (perpendicular orientation), vibrationally inelastic collisions would be associated more strongly with collisions involving these orientations. These collisions would produce weaker coupling to rotational excitation.

Dynamical corrugation is another previously reported effect that has relevance to the present study [26]. In that work, it was found that non-dissociative sticking of $\mathrm{CO}$ to $\mathrm{Cu}(100)$ was enhanced at normal incidence for high values of $E_{\mathrm{i}}$ and diminished at normal incidence for low values of $E_{\mathrm{i}}$. According to this picture, at high $E_{\mathrm{i}}$, rotational reorientation is not possible during the collision and the predominance of 'wrong orientation' collisions at high $E_{\mathrm{i}}$ leads to an enhanced effective corrugation of the surfaces. In the context of the present work, it is possible that only specific molecular orientations $(\theta, \varphi, x, y)$ lead to vibrational relaxation, whereas a much wider variety of orientations result in vibrationally elastic collisions. In this way, looking at the vibrationally sub-elastic channel might pre-select certain favorable orientations, essentially removing dynamical corrugation.

\section{Acknowledgement}

This work was supported by a grant from the Department of Energy Office of Basic Energy Sciences, Grant No. DEFG0203ER15441.

\section{References}

[1] C.T. Rettner, D.J. Auerbach, H.A. Michelsen, Phys. Rev. Lett. 68 (1992) 2547.

[2] A. Gross, W. Brenig, Surf. Sci. 289 (1993) 335

[3] G.A. Gates, G.R. Darling, S. Holloway, J. Chem. Phys. 101 (1994) 6281.

[4] A. Gross, W. Brenig, Chem. Phys. 177 (1993) 497.

[5] D.M. Newns, Surf. Sci. 171 (1986) 600.

[6] M. Head-Gordon, J.C. Tully, J. Chem. Phys. 103 (1995) 10137.

[7] C.T. Rettner, F. Fabre, J. Kimman, D.J. Auerbach, Phys. Rev. Lett. 55 (1985) 1904.

[8] E.K. Watts, J.L.W. Siders, G.O. Sitz, Surf. Sci. 374 (1997) 191.

[9] Y.H. Huang, C.T. Rettner, D.J. Auerbach, A.M. Wodtke, Science $290(2000) 111$.

[10] Y. Huang, A.M. Wodtke, H. Hou, C.T. Rettner, D.J. Auerbach, Phys. Rev. Lett. 84 (2000) 2985.

[11] A.M. Wodtke, Y. Huang, D.J. Auerbach, Chem. Phys. Lett. 364 (2002) 231

[12] S.M. Li, H. Guo, J. Chem. Phys. 117 (2002) 4499.

[13] J.T. Kindt, J.C. Tully, M. Head-Gordon, M.A. Gomez, J. Chem. Phys. 109 (1998) 3629.

[14] D.A. McCormack, G.J. Kroes, R.A. Olsen, J.A. Groeneveld, J.N.P. van Stralen, E.J. Baerends, R.C. Mowrey, Faraday Discuss (2000) 109.

[15] A.M. Wodtke, D.J. Auerbach, Y. Huang, Chem. Phys. Lett. 413 (2005) 326-330.

[16] H. Hou, C.T. Rettner, D.J. Auerbach, Y. Huang, S.J. Gulding, A.M. Wodtke, Faraday Discuss (1999) 181.

[17] Y.H. Huang, M. Sulkes, Rev. Sci. Instrum. 65 (1994) 3868.

[18] C. Amiot, J. Verges, Phys. Scr. 26 (1982) 422.

[19] C. Amiot, J. Mol. Spectrosc. 94 (1982) 150.

[20] R. Engleman, R.E. Rouse, H.M. Peek, V.D. Baiamonte, Los Alamos National Laboratory, Report No.: LA-4364, 1970.

[21] B. Berenbak, B. Riedmuller, C.T. Rettner, D.J. Auerback, S. Stolte, A.W. Kleyn . Physchemcomm (2001) art. no.-15.

[22] A.W. Kleyn, A.C. Luntz, D.J. Auerbach, Phys. Rev. Lett. 47 (1981) 1169

[23] G.D. Kubiak, J.E. Hurst, H.G. Rennagel, G.M. McClelland, R.N. Zare, J. Chem. Phys. 79 (1983) 5163.

[24] J. Kimman, C.T. Rettner, D.J. Auerbach, J.A. Barker, J.C. Tully, Phys. Rev. Lett. 57 (1986) 2053.

[25] C.T. Rettner, J. Kimman, D.J. Auerbach, J. Chem. Phys. 94 (1991) 734.

[26] J.T. Kindt, J.C. Tully, Surf. Sci. 477 (2001) 149. 